\title{
THE ANALYSIS TECHNIQUE OF SOCIAL MEDIA FOR DISASTER MANAGEMENT
}

\author{
SEONHWA CHOI \\ Safety Division, National Disaster Management Research Institute, South Korea.
}

\section{ABSTRACT}

Social media has transformed mass media based information traffic, and it has become a key resource for finding value in enterprises and public institutions. Particularly, with regard to disaster management, the necessity for public participation in policy development through the use of social media is emphasized. National Disaster Management Research Institute developed the Social Big Board, which is a system that monitors social Big Data in real time for the purpose of implementing social media disaster management. This real time monitoring system provides various information and insights based on the tweets, such as disaster issues, tweet frequency by region, original tweets, etc. The purpose of using this system is to take advantage of the potential benefits of social media in relation to disaster management. In this paper, Korean language text mining based Social Big Board will be briefly introduced, and disaster issue detection model, which is the key algorithms, will be described. The detection model of potential issues of these key algorithms is intensively defined and the performance of the models are compared and evaluated.

Keywords: big data, disaster issue detection, disaster management, social media.

\section{INTRODUCTION}

Disaster management in the Big Data generation, which is encountering major transformations can be viewed from two different contexts. First, it is capable of providing customized disaster information at the right time and the right place. In order to support the establishment of countermeasures and pre-emptive policies concerning complex and diverse disaster situations, it is necessary to go beyond the boundaries of related organizations and establish a foundation to incorporate the use of public opinions by expanding the scope to social, environmental, cultural, and economical areas for analysis [1]. Secondly, the information traffic channel is transitioning from mass media to social media. In the past, newspapers, TV, radio, and other such major media sources randomly delivered information to the public. Now, this delivery is transitioning to social media, which produces, consumes, and delivers the thoughts, ideas, and information to the public without discretion. Particularly, in the event of a disaster, social media acts as a medium to rapidly share precise disaster situations and information concerning evacuation. At the same time, it is also used as a medium to make communication possible on a national level with regard to disaster management.

Earthquakes in Haiti and Eastern Japan, flood in Gangnam in Korea, Asiana aircraft landing accident, recent Sewol Ferry capsizing, and numerous other disaster scenes are covered and shared over social media platforms, and the people responded by personally sharing information to minimize damages. In the United States, social media was used as a measure to provide a solution for the gas crisis caused by Hurricane Sandy (2012). Hurricane Sandy destroyed numerous power line towers and families were required to use power generators in their homes. As a result, it led to a gas crisis. In response, the people voluntarily shared gas station mapping information in real time over

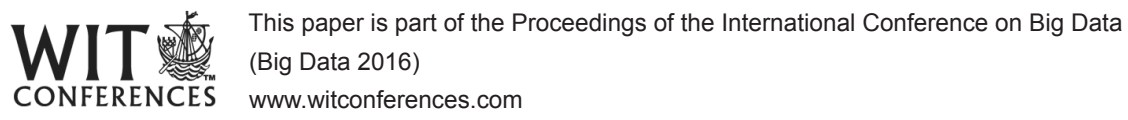

(C) 2016 WIT Press, www.witpress.com ISSN: 1755-7437 (paper format), ISSN: 1755-7445 (online), http://www.witpress.com/journals DOI: $10.2495 / \mathrm{DNE}-\mathrm{V} 11-\mathrm{N} 3-396-405$ 
facebook, twitter, and various other social media platforms [2, 3]. In other words, disaster response was implemented not by the government, but by the public. This example proved that the use of social media or communities in response to disasters is a new method of disaster management based on highly reliable collective intelligence. As we can see, social media, such as twitter, is used for fast information transmission in the event of a disaster, and it is also used as public method for sharing emergency information among the public [4]. Social media in the smart generation as a medium for reporting a disaster situation is faster than any other related organization, and is used as a channel to communicate with the public. Thus, it is necessary to establish a positive management measure with a focus on disaster management, and also to develop a social media monitoring technology.

In this paper, Social Big Board, which is a real time social big data monitoring system developed by the National Disaster Management Research Institute for purposes of disaster management, will be introduced, and disaster issue detection model, which is the key algorithms, will be described. Disaster issues are divided into two categories: potential issues, which refer to abnormal signs prior to disaster events, and occurrence issues, which is a notification of disaster events. The detection model of potential issues of these two categories is intensively defined and the performance of the models are compared and evaluated.

\section{SOCIAL BIG BOARD}

\subsection{Main features}

National Disaster Management Research Institute developed the Social Big Board is a system that monitors social Big Data in real time for purposes of implementing social media disaster management. Social Big Board collects a daily average of 36 million tweets in Korean in real time and automatically filters disaster safety related tweets. The filtered tweets are then automatically categorized into 71 disaster safety types. This real time tweet monitoring system provides various information and insights based on the tweets, such as disaster issues, tweet frequency by region, original tweets, etc. The purpose of using this system is to take advantage of the potential benefits of social media in relation to disaster management. It is a first step toward disaster management that communicates with the people and allows us to hear the voice of the people concerning disaster issues as well as to understand their emotions at the same time [5].

Social Big Board provides three main features. First, the purpose of real time social monitoring is to monitor disaster-specific issues on social networks in real time, and it categorizes 71 disaster safety types into five major categories (social disaster, human disaster, natural disasters, safety, and criminal activity). Main contents comprise original texts of recent tweets reloaded every minute, tweet frequency progress graph over the past 2 days, list of keywords extracted from tweets every hour, rate of disaster type frequency increase over a recent 2-day period compared to a previous 2-day period, and disaster issues showing rapid increase over the recent hour.

The second feature is real time issue analysis. It uses various search conditions on the 71 disaster types to provide support for a detailed analysis of the disaster types. It displays the frequency progress and original texts according to search conditions such as keywords, period, data format, etc.

The last feature is the social trend feature. It provides related word analysis and emotion analysis feature on the 71 disaster types. The social trend feature is provided based on the Big Data analysis engine, and it presents analysis results of the previous day's data. Related word refers to the keywords that are most frequently mentioned in tweets that have been classified as a disaster type. The keyword is first categorized into a relevant category from disaster management, related disaster, cause, damage, or related organization, and a category-specific related word analysis is performed to provide a related word map and the original tweet including the related words. Emotion analysis 
extracts words that represent emotions to provide a time-specific emotional progress and distribution map through a disaster type analysis. Eight emotions are categorized into positive or negative emotion groups, and it makes overall public emotion monitoring possible. Figure 1 shows a screenshot of the real-time monitoring service of Social Big Board.

\subsection{Disaster issue detection}

Social Big Board categorizes and defines disaster issues as potential issues and occurrence issues and applies an appropriate issue detection model. Potential issue analyzes tweets concerning signs of potential disasters prior to happening. In order to identify potential issues, non-uniform patterns in tweets must be identified, and identifying a trend is more important than accurate analysis. In addition, analysis on weather and social phenomenon must be conducted together with tweet frequency analysis. Another form of disaster issue is the disaster occurrence issue. This form notifies actual disaster events. It is different from potential issues in that it is more important to identify the first tweet concerning a disaster event. The accuracy of the contents and understanding the truth is the foremost priority. Therefore, frequency used in potential issues is not very important. It is necessary to identify the pattern of tweets notifying the disaster event [3].

The previously established potential issue model was based on the absolute frequency of tweets. If a keyword related to a specific disaster is identified in random tweets, it is categorized as the relevant disaster type and 1 is added to the frequency. The frequency is calculated for an hour, based on which a model is established and the potential disaster is determined. The existing frequency based model requires improvements because it puts only the number of tweets into consideration regardless of the contents of the tweet or risk factors.

\section{DISASTER ISSUE DETECTION MODEL IMPROVEMENT}

In order to improve the existing frequency based issue detection model, the rate of relevance and risk by disaster types was evaluated, and the performance was evaluated by establishing probability models capable of detecting issues by putting a higher weighted value on tweets with greater relevance and risk.

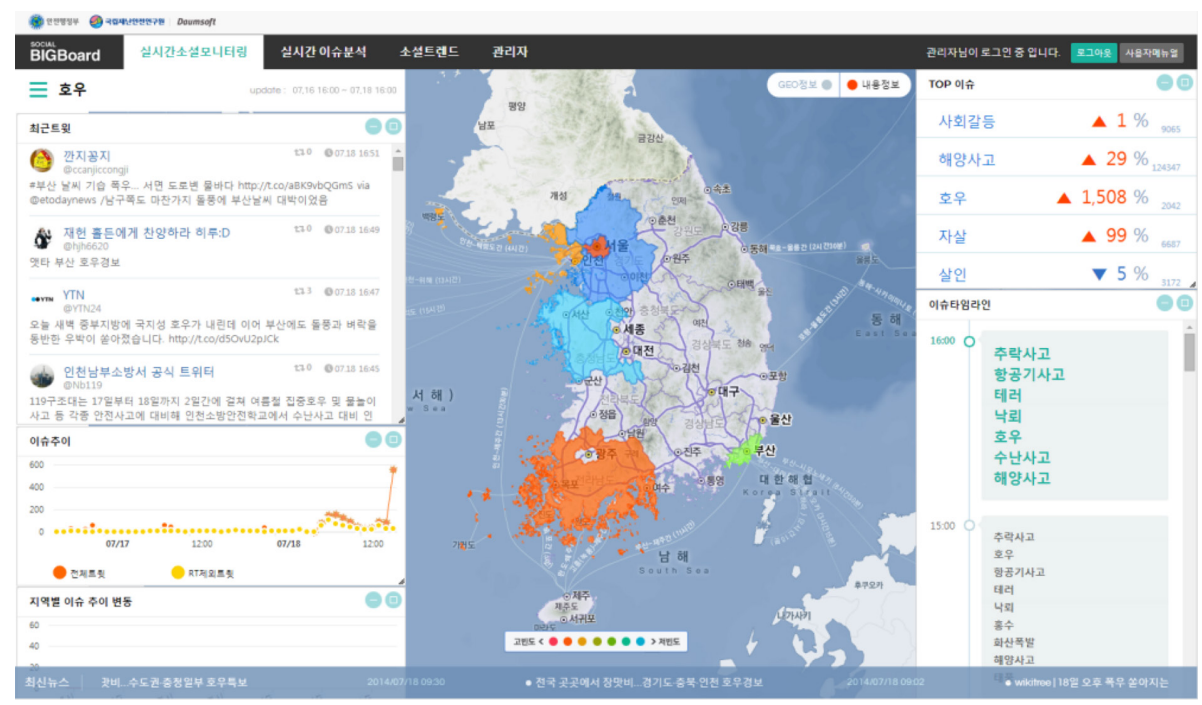

Figure 1: Real-time monitoring service screen. 
As a means to add more weighted value to keywords that appear frequently concerning specific disasters, the degree of relevance by keyword was quantified. The degree of relevance cannot reflect whether the tweet contains actual risk information because the disaster relevance is quantified based on frequency. Therefore, keywords containing damage risk are defined according to disaster types and the weighted value of the risk of each keyword is defined to establish an index to reflect the risk rate of each tweet and evaluate the issue detection model.

Table 1 shows tweets concerning yellow dust; the contents vary from plain information to contents expressing distress. In terms of disaster management, tweets expressing distress are more significant compared to plain tweets, and such tweets require differentiated detection compared to regular tweets. The risk assessment of a single tweet goes through three stages: First, the risk rate is studied based on machine learning to determine how much risk the keyword is expressing, then, the risk rate of the relevant tweet is evaluated based on the risk rate of the keywords included in the single tweet. Finally, the risk rate of all tweets are tallied according to disaster types to calculate the risk rate of the specific disaster.

\subsection{Relevance rate based potential issue detection model}

\subsubsection{Tweet relevance rate test and evaluation}

Disaster type specific tweet relevance rate adds a higher weighted value to keywords that frequently appear in tweets concerning specific disasters, and a relatively lower weighted value to keywords that do not quantify the relevance rate of tweets. The weighted value was defined using the rate of emergence of keywords in disaster-related tweets compared to regular tweets, and Chi Square and conditional probability method were used for each disaster type to study the weighted value and to compare the performance. For the Chi Square method, the weighted value of keyword $k$ is defined as $w_{\mathrm{k}}$ and formula $\frac{(a d-b c)^{2}(a+b+c+d)}{(a+b)(c+d)(b+d)(a+c)}$ was used for calculation. $a$ represents the number of disaster-related tweets containing the keyword $k . b$ represents the number of disaster-related tweets without the keyword $k$. $c$ represents the number of regular tweets with the keyword $k$, and $d$ represents the number of regular tweets without the keyword $k$. For conditional probability method, the weighted value of the keyword $k, w_{\mathrm{k}}$ was calculated using the following formula.

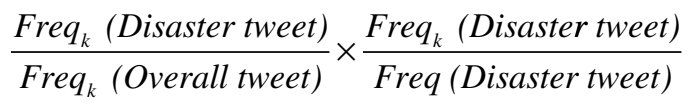

$$
\begin{aligned}
& \times \frac{\text { Freq }_{k}(\text { Disaster tweet })^{2}}{\text { Freq }_{k}(\text { Overall tweet }) \times \text { Freq }(\text { Disaster tweet })}
\end{aligned}
$$

Freq $_{k}$ (Disaster tweet):Number of disaster related tweets with keyword $k$

Freq $q_{k}$ (Overall tweet):Overall number of tweets with keyword $k$

Freq $_{k}$ (Disaster tweet):Number of tweets concerning relevant disaster

Table 1: 'Yellow Dust' related tweet examples.

\begin{tabular}{ll}
\hline Category & Tweet Example \\
\hline Regular tweet & 'Fine dust is very diligent, it doesn't even go to sleep' \\
Tweets with high number of related keywords & 'Is the sky cloudy or is it that there are as much fine dust as the clouds? \\
& $\begin{array}{l}\text { I can't even see the mountain in my neighborhood' } \\
\text { Tweets depicting hazardous situation }\end{array}$ \\
\hline
\end{tabular}


The weighted values of each keyword established through the learning process were used to calculate the rate of relevance of tweets by adding the weighted value of all keywords identified in the tweets, and also by dividing the sum of the weighted values of the keywords by the average rate of relevance per keyword, which is the number of keywords in the tweets. The performance of each method was evaluated. The test subjects included tweets from January 1st to June 16th of 2013, concerning railroad accidents, falling accidents, torrential rainfall, and nuclear radiation accident. The tweets were used to study the weighted values of keywords, and the weighted value and rate of relevance calculation methods were evaluated using tweets from June 17th of 2013. The evaluation was conducted using 30 tweets with highest relevance that have relevance to the disasters that actually occurred on that day, in other words, precision. As a result, the weighted value of keywords was calculated using the conditional probability method, and using the average rate of relevance per keyword showed the highest performance level (Table 2).

3.1.2 Relevance rate based potential issue detection model test and evaluation

In order to improve the existing potential issue detection model that uses discrete probability distribution model (Poisson's Distribution), continuous probability distribution models such as Normal distribution, Gamma distribution, and Weibull distribution models were used to evaluate the optimum model.

The overall rate of relevance of tweets with regard to a specific disaster type over a course of an hour is defined as the hourly relevance rate. In case a tweet concerning a specific disaster is not identified over a course of an hour, that is, if there are many instances of where the hourly relevance rate is 0 making it difficult to develop an adequate model for learning, daily peak from the hourly relevance rate is used to perform a probability modeling. Take a look at the probability modeling results illustrated in Fig. 2 with respect to the collapse accident, earthquake, and railroad accident. We can observe that the Weibull distribution is the most adequate probability distribution.

In the case of an earthquake, Gamma distribution and Weibull distribution both appear to be adequate in the probability density function. However, on observing the probability accumulation function it is apparent that Weibull distribution is far more adequate. This also applies to the railroad accident. Gamma distribution and Weibull distribution both appear to be adequate, but we can observe that Weibull distribution is more adequate compared to Gamma distribution. Therefore, it can be concluded that Weibull distribution model, which is capable of various probability models such as Normal distribution and exponential distribution, is most adequate for application on the potential issue model utilizing hourly relevance rate.

When using the potential probability model studied by disaster type if it shows that the aggregate probability value of random hourly relevance rate is above the threshold, it is determined as a potential issue and it is notified. The threshold in the existing model is fixed at $95 \%$, and anything above this value is determined as an issue.

Table 2: Evaluation results.

\begin{tabular}{lll}
\hline \multicolumn{1}{c}{ Weighted value } & Chi Square & $\begin{array}{l}\text { Conditional } \\
\text { Probability }\end{array}$ \\
\hline Rate of relevance & $103 / 120(0.8583)$ & $100 / 120(0.8333)$ \\
$\begin{array}{l}\text { Simple } \sum \text { (precision) } \\
\text { (precision) }\end{array}$ & $103 / 120(0.8583)$ & $106 / 120(0.8833)$ \\
$\begin{array}{l}\text { Simple } \sum / \log \text { (Tweet word frequency) } \\
\text { (precision) }\end{array}$ & $102 / 120(0.8500)$ & $102 / 120(0.8500)$ \\
\hline
\end{tabular}



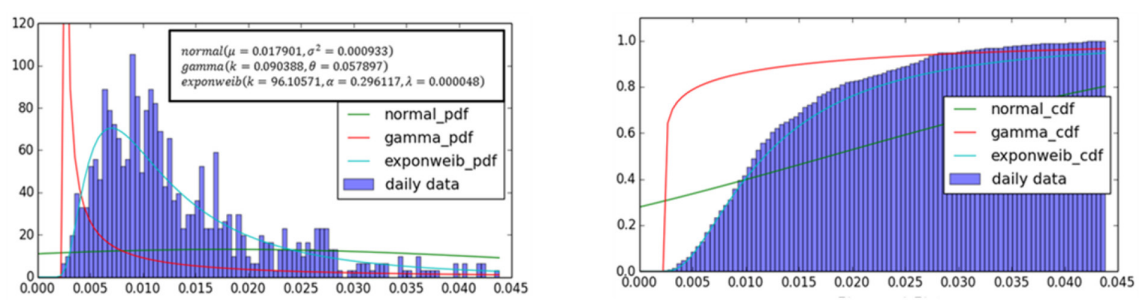

< Collapse accident >
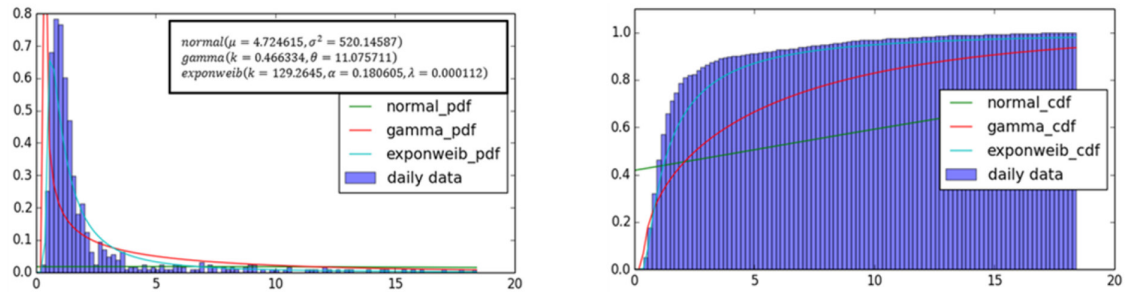

$<$ Earthquake >

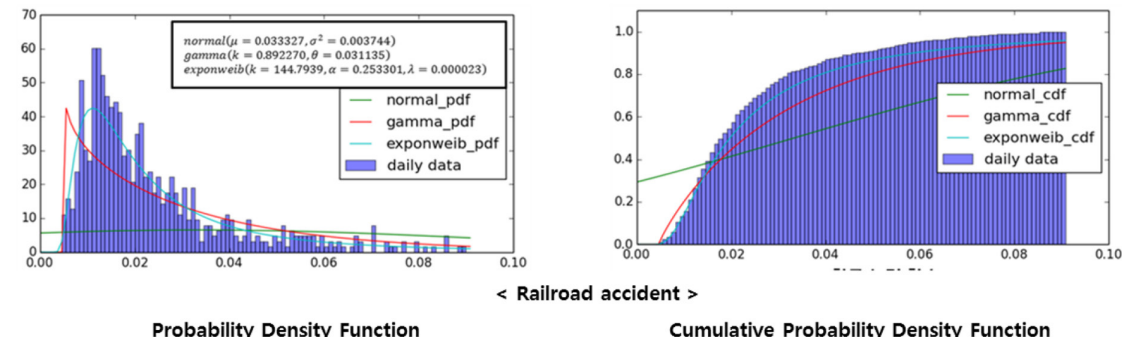

Figure 2: Disaster specific probability modeling results.

Optimum threshold value was set according to the disaster type specific probability model to verify the disaster issue detection performance. There are two types of thresholds: long term threshold (LTT) and short term threshold (STT), which are used to test the potential issue detection performance. LTT refers to the Weibull distribution aggregate probability threshold based on the daily peak hourly relevance rate taken 24 hours prior to the time of commencing the monitoring. STT refers to the percentile threshold with regard to the hourly relevance rate from between 50 hours to 1 hour prior to the time of detection.

In the event of a random disaster, twitter users have a tendency to post tweets concerning the disaster even after the disaster takes place. In such cases, using only LTT has a problem of recognizing the disaster as a potential disaster even after the disaster has taken place. Thus, STT was developed to supplement LTT. In order to evaluate the optimum combination of LTT and STT, Precision, Recall, $\mathrm{F}_{1}$-Measure, and $\mathrm{F}_{2}$-Measure were applied. $\mathrm{F}_{1}$ means the performance when the weighted value of precision and recall are set equally, whereas $F_{2}$ is the performance when doubling the weighted value of recall compared to precision. $\mathrm{F}_{2}$ performance is used as an important reference because recall is relatively more important than precision in terms of detecting disaster issues.

$$
F_{1}=2 \times \frac{\text { recall precision }}{\text { recall }+ \text { preciaion }}
$$




$$
F_{2}=\left(1+2^{2}\right) \times \frac{\text { recall precision }}{\text { recall }+\left(2^{2} \text { preciaion }\right)}
$$

Tweet data concerning collapse accidents, earthquakes, and railroad accidents from January to September of 2014 were used as the test data. Disaster-related news were directly reviewed by people to identify disaster issues and develop a set of correct answers, and 3 hours after the occurrence of the disaster issue was assumed as the correct answer. As a result of the evaluation, $\mathrm{F}_{2}$ performance was greatest when LTT is 85 and STT is 95 for collapse accidents, when LTT is 65 and STT is 95 for earthquakes, and when LTT is 75 and STT is 95 for railroad accidents. Table 3 illustrates the potential issue determination performance comparison for cases applying a fixed threshold of 95 on existing frequency based Poisson's distribution model and for cases applying a disaster type specific LTT and STT combination of the highest $\mathrm{F}_{2}$ performance as the threshold on a relevance rate based Weibull distribution model. In comparison to applying a fixed threshold to the existing frequency based model, applying a disaster-specific threshold to the relevance rate based model showed greater performance in all disaster type tests.

\subsection{Risk rate based potential issue detection model}

3.2.1 Risk rate test and evaluation

Machine learning method was used for the weighted value reflecting the risk rate of all keywords. It is necessary to add a greater weighted value to keywords that appear frequently in tweets that notify potential risk compared to keywords that appear in ordinary tweets.

Table 3: Potential issue detection model threshold test results.

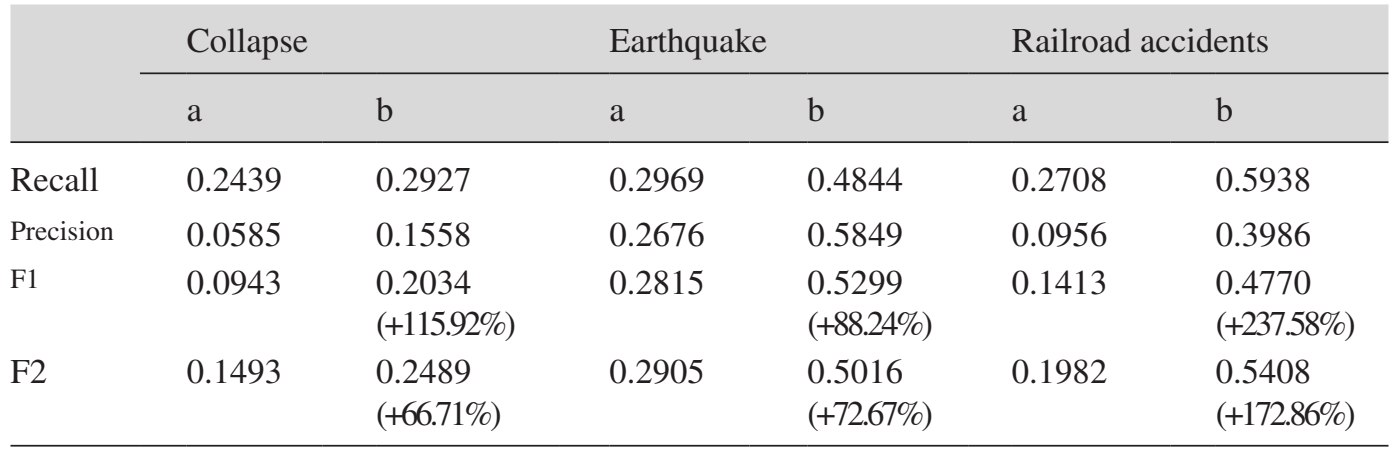

a : Frequency, b: Relevance rate

Table 4: 'Land subsidence' disaster related risk/non-risk tweet examples.

\begin{tabular}{ll}
\hline Category & Tweet example \\
\hline $\begin{array}{l}\text { Tweets depicting potential } \\
\text { risk }\end{array}$ & $\begin{array}{l}\text { 'Sinchon-Gangnam sinkhole... main cause is deterioration of } \\
\text { sewage pipes in subway lines...' }\end{array}$ \\
$\begin{array}{l}\text { Tweets depicting potential } \\
\text { risk }\end{array}$ & 'Walkway surface in front of Sinwol4dong unit 434-6 is uneven \\
Regular tweets & and dangerous due to cold weather.' \\
Regular tweets & 'Arghhh... Whenever I hear stories about sinkholes in Seoul... $\mathrm{T} T$ \\
\hline
\end{tabular}


As shown in Table 4, words such as 'deterioration,' 'uneven', and 'dangerous' are keywords that notify potential risk, and such words are frequently found in tweets. In this study, a learning set is formed by dividing tweet sets that notify potential risk and those that do not, and risk rate weighted value were added for each keyword through machine learning. The risk rate weighted value added to each keyword is used to quantify the risk rate of each tweet, and the learning process is as shown below.

1. Initial learning set establishment: Extract tweets that satisfy specific conditions and tweets containing risk information and those that are not categorized by people.

2. Weighted value learning: Maximum Entropy (ME) model based risk rate weighted value learning

3. Weighted value relearning: Weighted value learned through 2. is used to categorize large quantity of tweets, and the categorized tweets are used for relearning weighted values.

4. Preparatory establishment of risk rate weighted value.

Machine learning shows better performance with greater number of learning sets, but it is difficult for human hands to establish numerous amounts of learning sets. Therefore, the initial learning sets established by human hands are used as subjects to learn models, and the learned models are used to categorize tweets, which are then used to relearn the risk rate weighted value to establish the final risk rate weighted value index.

The initial learning set is developed by extracting 8,913 random tweets from January 1st 2013 to December 31 st 2014 . The tweets were manually categorized by people. The initial learning set categorized by human hands was used as the subject to learn the model, based on which potential risk tweets and non-risk tweets were categorized. The categorized tweets were then used to develop a relearning set. The relearning set comprises roughly 400 times more tweets compared to the initial learning set.

In order to supplement the inaccuracy of the relearning set developed through automated categorization, tweets with high risk rate weighted value and low risk rate weighted value were included in the learning set. In order to define the standard for high risk rate tweets and low risk rate tweets, an answer set consisting of 10,000 tweets manually categorized by human hands were used as the subject to differentiate the high/low risk rate weighted value ratio. As a result of evaluating the accuracy of categorization, the model that used top $1 \%$ and low $8 \%$ risk rate weighted value tweets from the relearning set showed the highest performance level. Eventually, top 1\% and low $8 \%$ of the tweets sorted by risk rate from 3,689,806 relearning sets were used to relearn the model and establish a risk rate weighted value index for the keywords.

The rate of risk was converted to z-score to create a normal distribution, and exponential function was used to convert all the risk rates to positive numbers. In other words, the risk rate of individual risk rates is calculated using the following formula.

$$
\text { Danger_Weight(tweet })=2^{\frac{x-\mu}{\sigma}}
$$

In the above formula, $x$ represents the risk rate of individual tweets, $\mu$ represents the average risk rate of learning set tweets, and $\sigma$ represents standard deviation.

3.5 Risk rate based potential issue detection model test and evaluation

Disaster type specific hourly risk rate is used as the overall risk rate of tweets concerning specific disasters as the grounds to determine whether the specific disaster is an issue requiring attention. 
Table 5: Relevance rate and risk rate based issue detection test results.

\begin{tabular}{|c|c|c|c|c|c|c|}
\hline & \multicolumn{2}{|c|}{ Collapse } & \multicolumn{2}{|c|}{ Earthquake } & \multicolumn{2}{|c|}{ Railroad accident } \\
\hline & $a^{*}$ & $b^{*}$ & $a^{* *}$ & $b^{* *}$ & $a^{* * *}$ & $\mathrm{~b}^{* * * *}$ \\
\hline Recall & 0.2927 & 0.3170 & 0.4844 & 0.5649 & 0.5938 & 0.6042 \\
\hline Precision & 0.1558 & 0.5000 & 0.5849 & 0.4458 & 0.3986 & 0.4265 \\
\hline $\mathrm{F} 1$ & 0.2034 & $\begin{array}{c}0.3880 \\
(+90.76 \%)\end{array}$ & 0.5299 & $\begin{array}{c}0.4983 \\
(-5.96 \%)\end{array}$ & 0.4770 & $\begin{array}{c}0.5000 \\
(+4.82 \%)\end{array}$ \\
\hline F2 & 0.2486 & $\begin{array}{c}0.3421 \\
(+37.44 \%)\end{array}$ & 0.5016 & $\begin{array}{c}0.5362 \\
(+6.90 \%)\end{array}$ & 0.5408 & $\begin{array}{c}0.5577 \\
(+3.13 \%)\end{array}$ \\
\hline
\end{tabular}

a: Relevance rate, b: Risk rate

Risk rate based potential issue detection model was established and evaluated using the same methods used for relevance rate based model establishment and evaluation.

Disaster related tweet data concerning collapse accidents, earthquakes, and railroad accidents from January 1st 2014 to September 30th 2014 were used as the test data. With regard to the answer set, disaster-related news articles were manually reviewed to extract disaster events, and 3 hours from the time when the disaster event occurred was assumed to be the time of disaster issue occurrence. The following table is a performance comparison of relevance rate based disaster issue detection model and risk rate based model.

In Table $5 \mathrm{a}^{*}$ represents LTT 85 and STT 95 after modeling the daily peak hourly relevance rate data using Weibull distribution. $\mathrm{a}^{* * *}$ represents LTT 65 and STT 95, whereas $\mathrm{a}^{* * *}$ represents LTT 75 and STT 95 . b* represents LTT 95 and STT 95 after modeling the daily peak hourly relevance rate data using Weibull distribution. $\mathrm{b}^{* *}$ represents LTT 60 and STT 90, whereas $\mathrm{b}^{* * *}$ represents LTT 65 and STT 95.

Collapse accident and railroad accident disaster test results showed that risk rate based model is more superior in performance compared to the existing relevance rate based model. Earthquake disaster type $\mathrm{F}_{1}$ test results showed that risk rate based model is inferior in performance compared to the existing relevance rate based model. However, in contrast, $\mathrm{F}_{2}$ test results showed that risk rate based model is superior in performance compared to the existing relevance rate based model.

\section{CONCLUSION}

In this paper, Korean language text mining based Social Big Board was briefly introduced, and the disaster issue detection model and emotional quotient quantification method were described. Disaster issues are divided into two categories: potential issues, which refers to abnormal signs prior to disaster events, and occurrence issues, which is a notification of disaster events. Each detection model was defined and the performance of each model was compared and evaluated. Risk rate based model, which puts a greater weighted value on tweets with contents that notify the potential risk of a disaster, showed highest performance for potential issue detection model.

By using our system to manage disasters, we can expect remarkable changes in disaster management: not just predicting a simple hazard based on the structured data but sensing and analyzing signs using social data as well as the structured disaster data. We have a long way yet to put this system to practical use. In the future, by developing the integrated analysis between structured data and unstructured data, namely big data, an intelligence disaster response system will be possible. 


\section{REFERENCES}

[1] Choi, S.H., The model for sensing of disaster signs based on big data. Journal of Korean Society of Hazard Mitigation, 14(2), pp. 149-157, 2014 (In Korean). http://dx.doi.org/10.9798/KOSHAM.2014.14.2.149

[2] Choi, S.H., The model for sensing of disaster signs based on big data. Proceeding of the International Conference on CAIPT, pp. 169-172, 2013.

[3] Seon, H.C. \& Byung, G.B., Disaster issue detection model from social big data. Information Science Society Journal, 20(5), pp. 286-290, 2014 (In Korean).

[4] Jeannette, S., Leysia, P. \& Irina, S., Backchannels on the front lines: emergent uses of social media in the 2007 Southern California Wildfires. Proceedings of the 5th International ISCRAM Conference, Washington DC., USA, 2008.

[5] Seon, H.C., Jong, G.L. \& Woon, G.Y., Disaster management competnece reinforcement for self-governing bodies using big data. Local Administration, 730, pp. 16-19, 2014 (In Korean).

[6] Tsolmon, B., Kwon, A.R. \& Lee, K.S., Extracting social events based on timeline and sentiment analysis in twitter corpus. Natural Language Processing and Information Systems LNCS, 7337, pp. 265-270, 2012. http://dx.doi.org/10.1007/978-3-642-31178-9_32

[7] Seon, H.C., Disaster issue detection model from disaster relevance rate based social big data. Academic Conference Journal of Korea Information Processing Society, pp. 829-832, 2014 (In Korean).

[8] The Urban Geographies of Hurricane Sandy in New York City, floating sheep, available at http://www.floatingsheep.org/2012/10/the-urban-geographies-of-hurricane.html

[9] Hurricane Sandy and the Geographies of Flooding on Twitter, floating sheep, available at http://www.floatingsheep.org/2012/10/hurricane-sandy-and-geographies-of.html 